\title{
Design of a Distributed Signal Processing Unit for Transmission Line Protection in a Centralized Substation Protection Architecture
}

\author{
$1^{\text {st }}$ Fabian Hohn \\ Electric Power \& Energy Systems \\ KTH Royal Institute of Technology \\ Stockholm, Sweden \\ fhohn@kth.se
}

\author{
$2^{\text {nd }}$ Jianping Wang \\ Electric Power \& Energy Systems \\ KTH Royal Institute of Technology \\ Stockholm, Sweden \\ jiawan@kth.se
}

\author{
$3^{\text {rd }}$ Lars Nordström \\ Electric Power \& Energy Systems \\ KTH Royal Institute of Technology \\ Stockholm, Sweden \\ larsno@kth.se
}

\begin{abstract}
Travelling-wave and time-domain-based protection functions provide significant response time improvements over conventional phasor-based protection functions in power systems. These types of protection functions require very high sampling rates in the order of several hundreds of $\mathrm{kHz}$. This paper proposes a novel centralized substation protection architecture (CPC) based on distributed signal processing units (DSPU) that enables the deployment of these high sampling rate applications in digital substations utilizing an Ethernet-based process-level network. The design of the DSPU is elaborated, and its signal processing algorithms are discussed. Moreover, the performance of the DSPU is analysed through dynamic tests and verified through a numerical electromagnetic transient simulation.

Index Terms-Digital signal processing, Distributed processing, Edge computing, Power system protection, Power system transients, Substation protection, Systems architecture
\end{abstract}

\section{NOMENCLATURE}

$x(t) \quad$ Continuous-time function, $t \in \mathbb{R}$.

$x[n] \quad$ Discrete-time quantized sequence, $n \in \mathbb{Z}$.

$x_{d_{i}}\left[n_{d_{i}}\right] \quad$ Downsampled sequence by a factor $d_{i}$.

$d_{i}$

$n_{d_{i}}$

$F_{N}$

$f_{N, d_{i}}$

$F_{S}$

$f_{s, d_{i}}$

$M_{i}$

$f_{p, i}$

$f_{s t, i}$

$\delta_{p, i} / \delta_{s t, i}$

$\tau_{i}$

$e_{k, i}$

$X_{d_{i}}^{n c}[m ; k]$

$X_{d_{i}}[m ; k]$

$m$

$f_{k, m}$

$L_{H, m}$

$B_{H, m}$

$P_{H}$

$t_{H, m}$
Downsampling factor at $i^{\text {th }}$ decimation stage. Sample index after $i^{\text {th }}$ decimation stage.

Nyquist frequency of the signal $x(t)$.

Nyquist frequency of the signal $x_{d_{i}}\left[n_{d_{i}}\right]$.

Sampling frequency of ADC.

Digital sampling rate after $i^{\text {th }}$ decimation stage. Number of taps of the $i^{\text {th }}$ FIR lowpass filter.

Passband edge of the $i^{\text {th }}$ FIR lowpass filter.

Stopband edge of the $i^{\text {th }}$ FIR lowpass filter.

Ratio of passband to stopband error.

Group delay of the $i^{\text {th }}$ FIR lowpass filter.

Magnitude error of $k^{\text {th }}$ harmonic at $i^{\text {th }}$ FIR

Not-compensated $k^{\text {th }}$ spectral component.

Compensated spectral $k^{\text {th }}$ component.

Indicates the latest sample of STFT window.

Estimated frequency of $k^{\text {th }}$ component.

Window length of discrete STFT.

Overlap of discrete STFT window.

Cycle time of harmonic module.

Timestamp of synchronised phasors.

\section{INTRODUCTION}

Digital signal processing (DSP) plays a vital role in power system protection for extracting different features of the voltage and current signals such as the fundamental phasor components, RMS values or the $2^{\text {nd }}$ to $5^{\text {th }}$ harmonic components. The importance of DSP in protection systems will increase due to power system phenomena such as increased magnitudes of harmonics and interharmonics [1], in particular subharmonics, as well as reduced system inertia and reduced short circuit ratio (SCR) [2]. These phenomena are mainly caused by an increasing number of inverter-connected generation and other non-linear loads. Travelling waves along transmission lines are fault induced signals and therefore provide a promising solution for protection systems in low SCR power systems. In [3] a transient based protection scheme is tested based on a sampling rate of 80 samples per cycle as suggested in IEC 61850-9-2LE. Nevertheless, higher sampling rates are required for travelling-wave based differential protection schemes. In [4] a sampling rate of $1 \mathrm{MHz}$ is proposed. These high sampling rates impose particular challenges for digital substations utilising an Ethernet-based process-level network and having a high degree of functional integration on a single platform consisting of computing hardware and execution environment. This type of architecture is referred to as centralized substation protection and control (CPC) as specified in [5] and is expected to foster new centralized functions such as dynamic state estimation-based protection schemes [6] at the substation level. Most of the proposed CPC architectures use merging units (MUs) to publish sampled values (SV) of the current and voltage signals on the process bus as in [7] and in [8], while the digital signal processing (DSP) algorithms and protection logic are executed on the centralized platform. This approach has two drawbacks. Firstly, publishing the signals with high sampling rates increases the communication burden significantly. Maximum latency and high bandwidth requirements decrease the size of process-level networks based on highavailability seamless redundancy (HSR) as indicated in [9]. Secondly, all the computationally expensive DSP algorithms, 
such as finite impulse response (FIR) lowpass (LP) filter, shorttime fourier transform (STFT), and discrete wavelet transform (DWT), are executed on the centralized platform, which leads to high computational requirements in order to execute the protection functions in real-time. In order to mitigate the first challenge of a high communication burden, [10] proposes to implement the travelling wave feature extraction, such as polarity and magnitude, on the MU and then publish the results on the process bus. The IEEE standard about "Recommended Practice for Signal Treatment Applied to Smart Transducer" indicates that signals will be entirely processed at the point of measurement in the future [11]. This recommendation is picked up by [12] and is applied to the application of power system protection in substations. The paper [12] proposes to integrate all the required DSP algorithms of the protection function in a dedicated device termed a distributed signal processing unit (DSPU), which publishes the results of the DSP algorithms on the process bus instead of raw samples. Thus the high communication burden as well as high computational burden of the centralized platform can be lowered and a scalable CPC architecture can be obtained.

\section{A. Scope of the Paper}

This paper builds on the CPC architecture proposed in [12] and modifies it in order to emphasise the importance of having a DSPU for each protection object such as transformer, transmission line or power system bus. Furthermore, this paper discusses the design of the different DSP algorithms required for transmission line protection.

\section{B. Outline of the Paper}

The reminder of this paper is structured as follows. In Sec. II the requirements for digital substations are stated and the novel CPC architecture is described. Then in Sec. III the design and structure of the transmission line DSPU and its DSP algorithms are elaborated. Furthermore, Sec. III discusses in detail the multirate filter bank and the discrete STFT utilised in the transmission line DSPU. In Sec. IV the functional performance of the multirate filter bank and discrete STFT is evaluated through different dynamic tests as well as through an electromagnetic transient (EMT) simulation. Lastly, Sec. V concludes the paper and proposes future work.

\section{Substation Automation Architectures}

This section discusses the requirements that have been considered in this paper during the design of the DSPU. Then the novel CPC architecture based on DSPUs is described.

\section{A. Requirements for Protection Systems in Substations}

The two parts of IEC standards 61850-5 and 61869-9 comprise the communication requirements for protection functions and the requirements for digital interfaces of instrument transformers, respectively. Moreover, the IEEE standard 37.118 for Synchrophasor Measurements for Power Systems has been considered. Nonetheless, it should be mentioned that the main application of IEEE 37.118 is power system operation and
TABLE I

REQUIREMENTS FOR PROTECTION SYSTEMS IN SUBSTATIONS

\begin{tabular}{c|c|c} 
Requirements & Value & Source \\
\hline \hline Transfer-time of SV & $3 \mathrm{~ms}$ & IEC61850-5 [13] \\
\hline Processing delay of SV & $2 \mathrm{~ms}$ & IEC61869-9 [14] \\
\hline Time synchronization accuracy & $1 \mu \mathrm{s}$ & IEC61850-5 [13] \\
\hline Ratio error at $16.7 \mathrm{~Hz}$ or $20 \mathrm{~Hz}$ & $10 \%(0.92 \mathrm{~dB})$ & IEC61869-6 [15] \\
\hline Ratio error at $2^{\text {nd }}$ to $5^{\text {th }}$ harm. & $10 \%(0.92 \mathrm{~dB})$ & IEC61869-6 [15] \\
\hline Inst. error from $50 \mathrm{~Hz}$ to $50 \mathrm{kHz}$ & $10 \%(0.92 \mathrm{~dB})$ & IEC61869-6 [15] \\
\hline Instantaneous error at $500 \mathrm{kHz}$ & $30 \%(3 \mathrm{~dB})$ & IEC61869-6 [15] \\
\hline Anti-aliasing filter attenuation & $\geq 20 \mathrm{~dB}$ & IEC61869-6 [15] \\
\hline Total vector error (TVE) & $1 \%$ & IEEE37.118 [16]
\end{tabular}

analysis and not power system protection in substations. Tab. I summarises the requirements that are of major concern for CPC architectures.

\section{B. Proposed CPC Architecture based on DSPU}

The proposed CPC architecture suggests to use a dedicated DSPU per protection object at the process level, which contains the DSP algorithms required by the corresponding protection functions, such as distance, differential or overcurrent protection based on phasor, time-domain or travelling-wave quantities. The protection object is defined by its respective protection zone with the current transformers (CTs) acting as boundaries [17]. This paper has modified the CPC architecture proposed in [12] and emphasises the importance of having a DSPU for each protection object such as transformer or power system bus. This modification is required, since some of the protection functions are partly based on the differential current samples instead of pre-processed phasor-domain quantities. The DSPU uses the three-phase secondary current and voltage signals as input and publishes the results of the DSP algorithms on an HSR closed-loop network at a synchronised publishing rate. Synchronisation is achieved through the precision time protocol (PTP) utilising the power utility profile and hardware timestamping to obtain an accuracy of below $1 \mu$ s. Since the publishing rate is independent of the sampling rate, the communication load is reduced in case of high sampling rate protection applications. The DSP results are encoded using the common data classes (CDC) defined in IEC 61850-7-3 and are published by the Generic Object-Oriented Substation Event (GOOSE) application layer protocol configured with a fixed and synchronised publishing rate of $1 \mathrm{~ms}$, similar to [18] for wide-area networks (WAN). Furthermore, the entire protection function logic of the substation is integrated on a centralized platform, as shown in Fig. 1, and executed with a cycle time of $1 \mathrm{~ms}$ or slower. It can be seen from Fig. 1 that the proposed CPC architecture might lead to increased copper cabling in comparison to the MU approach. This is caused by the fact that the DSPU for transformer protection and system bus protection uses current and voltage signals from multiple bays, whereas the MU uses only measurements from its respective bay. Nevertheless, this drawback is expected to be overcome through the increased technical readiness and deployment of optical instrument transformers [19] and Rogowski Coils [20]. 


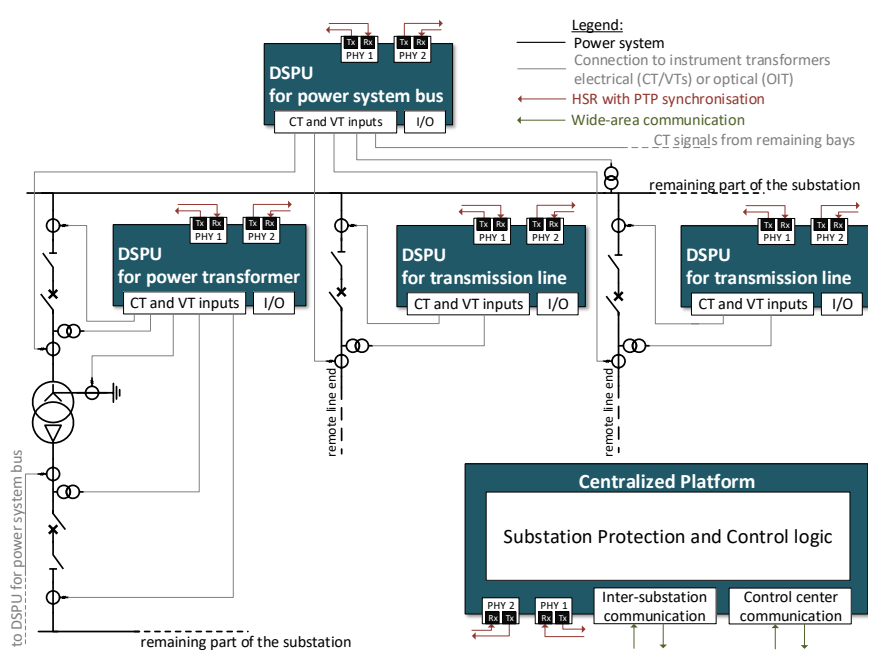

Fig. 1. Proposed CPC architecture based on DSPU

\section{DSPU FOR TRANSMISSION LINE PROTECTION}

The following section describes the signal processing structure of the DSPU and then focuses on the multirate filter bank and the discrete STFT.

\section{A. Signal Processing Structure of the DSPU}

The different protection functions deployed in the power system can be clustered according to the required frequency spectrum of their input signals, namely phasor based, timedomain based and travelling-wave based. Most of the currently deployed protection functions are phasor based, which means that the fundamental component is of major importance as well as the $2^{\text {nd }}$ to $5^{\text {th }}$ harmonics for the detection of transformer inrush conditions and overexcitation situations, respectively. These quantities are provided by the Harmonic module as depicted in Fig. 2. Moreover, the time-domain protection functions are mostly concerned with the incremental quantities of the current and voltage signals that are obtained using the superposition principle. Furthermore, different time-domain functions utilise waveshape analysis to increase the security of phasor based protection functions. These quantities are provided by the time-domain protection module and waveshape analysis module as shown in Fig. 2. The third group of protection function is travelling-wave based and analyses the electromagnetic waves of the power system transients in order to extract information about the polarity or amplitude as well as timing information. Thus the high frequency components of the voltage and current signals are of importance. These quantities are provided by the travelling-wave protection module. Lastly, the Interhamonic module is depicted in Fig. 2. Its main purpose is to provide information about the occurrence of interharmonics, such as subsynchronous resonances. The parameter $P_{x x}$ underneath the modules indicates the cycle time of the corresponding module. It can be seen that the Interhamonic module publishes its DSP results every $1 \mathrm{~s}$ whereas all the other modules report their results every $1 \mathrm{~ms}$. The 1 second cycle time of the Interhamonic module has been

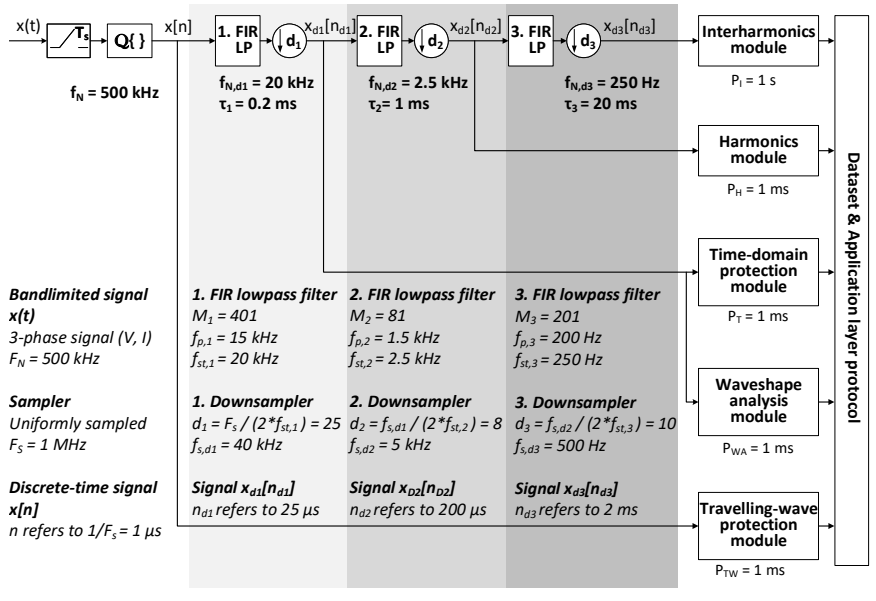

Fig. 2. Signal processing structure of the DSPU for transmission lines

chosen in order to obtain a high frequency resolution of the results. Due to the different frequency spectrum requirements of the DSP algorithms, a multirate filter bank consisting of FIR LP filters is used to supply the signal inputs with the required frequency spectrum to the respective DSP modules as shown in Fig. 2. The input signals of the DSPU are the three-phase continuous functions $x(t)$ of secondary currents and voltages, which have been band-limited by analog antialiasing filters to the Nyquist frequency $F_{N}=500 \mathrm{kHz}$. Thus the band-limited function $x(t)$ can be uniformly sampled with a sampling frequency $F_{S}=1 \mathrm{MHz}$ and the discrete-time quantized signal $x[n]$ is obtained as shown in (1).

$$
x[n]=Q\left(x\left(n T_{S}\right)\right)
$$

Where the sampling period $T_{S}$ is defined in (2) and $Q(x)$ represent the quantization process, which adds a quantisation error to the signal.

$$
T_{S}=\frac{1}{F_{S}}=1 \mu \mathrm{s}
$$

\section{B. Multirate Filter Bank}

After the sampling and quantization processes the sequence is filtered by three cascaded causal FIR LP filter and downsamplers. Since the synchronization of samples and of measurements is of high importance in protection systems, FIR filters have been chosen over infinite impulse response (IIR) filters due to their linear phase response in the passband. Additionally, causal FIR filters are Bounded-Input-BoundedOutput (BIBO) stable, since the impulse response is absolutely summable. Equation (3) shows the discrete convolution in the form of a constant-coefficient-difference equation (CCDE) of the $i^{\text {th }}$ causal FIR filter and the succeeding downsampler. 
TABLE II

DESIGN PARAMETERS FOR THE THREE FIR LP FILTERS

\begin{tabular}{c|c|c|c} 
Design parameters & $\mathbf{1}^{\text {st }}$ FIR LP & $\mathbf{2}^{\text {nd }}$ FIR LP & $\mathbf{3}^{\text {rd }}$ FIR LP \\
\hline \hline Number of taps $M_{i}$ & 401 & 81 & 201 \\
\hline Passband edge $f_{p, i}$ & $15 \mathrm{kHz}$ & $1.5 \mathrm{kHz}$ & $200 \mathrm{~Hz}$ \\
\hline Stopband edge $f_{s t, i}$ & $20 \mathrm{kHz}$ & $2.5 \mathrm{kHz}$ & $250 \mathrm{~Hz}$ \\
\hline Weight $\delta_{p, i} / \delta_{s t, i}$ & $2 / 1$ & $2 / 1$ & $2 / 1$
\end{tabular}

$$
\begin{aligned}
x_{d_{i}}\left[n_{d_{i}}\right] & =\sum_{k=0}^{M_{i}-1} h_{i}[k] x_{d_{(i-1)}}\left[n_{d_{i}} d_{i}-k\right] \\
& =\sum_{k=0}^{M_{i}-1} b_{i, k} x_{d_{(i-1)}}\left[n_{d_{i}} d_{i}-k\right], i \in \mathbb{N}^{+}
\end{aligned}
$$

Where $h_{i}$ is the impulse response of the $i^{\text {th }}$ FIR filter and with the $k^{\text {th }}$ coefficients corresponding to $h_{i}[k]=b_{i, k}$. The sequence before the first decimation stage is given by $x_{d_{0}}\left[n_{d_{0}}\right]=x[n]$.

In order to find the filter coefficients $b_{i, k}$ the Parks-McClellan algorithm has been used, which yields an equiripple magnitude response with linear phase and which minimizes the maximum error in the pass- and stopbands [21]. Tab. II contains the filter design parameters used as an input to the algorithm and Fig. 3 shows the frequency response of the three different FIR LP filters. The passband edge $f_{p, i}$ and stopband edge frequencies $f_{s t, i}$ have been chosen under the consideration of the required Nyquist frequency $f_{N, d_{i}}$ by each of the DSP modules. Then the number of taps has been selected considering the maximum error and processing delay requirements as well as the minimum attenuation requirements stated in Tab. I. It can be seen from Fig. 3 that there is a margin between the requirements and the filter performance that allows a further reduction of the high number of taps and thereby decreases the computational burden in terms of multiply-accumulate operations. The number of taps defines the group delay $\tau_{i}$ of the $i^{\text {th }}$ filter given in (4).

$$
\tau_{i}=\frac{M_{i}-1}{2} \frac{1}{f_{s, d_{(i-1)}}}, i \in \mathbb{N}^{+}, f_{s, d_{0}}=F_{S}
$$

Where the digital sampling rate $f_{s, d_{i}}$ and the downsampling factor $d_{i}$ are given in 5 and 6 , respectively.

$$
\begin{gathered}
f_{s, d_{i}}=\frac{f_{s, d_{(i-1)}}}{d_{i}}, i \in \mathbb{N}^{+}, f_{s, d_{0}}=F_{S} \\
d_{i}=\frac{f_{s, d_{(i-1)}}}{2 f_{s t, i}}, i \in \mathbb{N}^{+}, f_{s, d_{0}}=F_{S}
\end{gathered}
$$

The downsampling factor $d_{i}$ is conservatively chosen in (6) in order that the Nyquist frequency $f_{N, d_{i}}$ equals the stopband edge frequency $f_{s t, i}$. Evaluating (4) for the $1^{\text {st }}, 2^{\text {nd }}$ and $3^{\text {rd }}$ FIR LP filter, the group delays of $200 \mu \mathrm{s}, 1 \mathrm{~ms}$ and $20 \mathrm{~ms}$ are obtained, respectively. Adding the group delays of the $1^{\text {st }}$ and $2^{\text {nd }}$ multirate stage yields a total delay of $1.2 \mathrm{~ms}$ that allows the time-domain protection module, waveshape analysis module
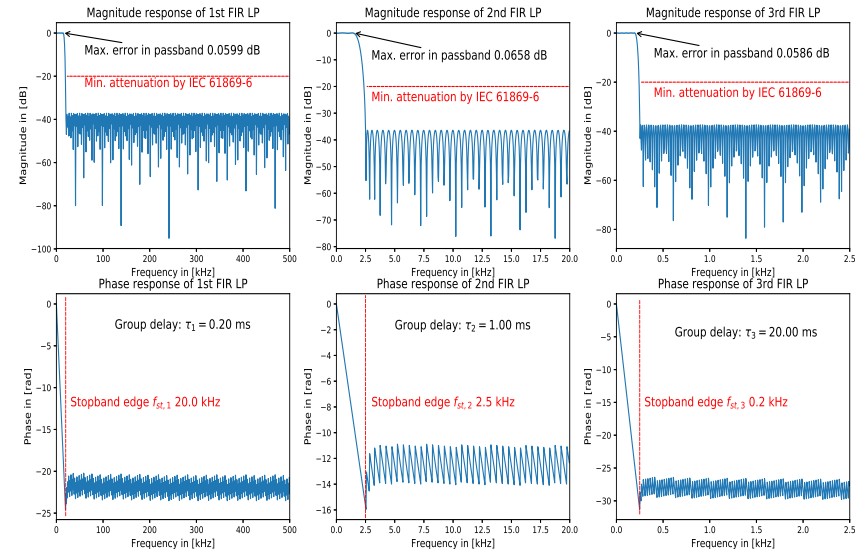

Fig. 3. Frequency response of the FIR lowpass filters

and harmonic module to process the input sequence faster than the maximum allowed processing delay requirement of $2 \mathrm{~ms}$ for SV.

\section{Harmonic Module}

The purpose of the harmonic module is to obtain synchronised phasors of the harmonic components of interest for the transmission line protection. At its core the discrete STFT is applied on the sequence $x_{d_{2}}\left[n_{d_{2}}\right]$ for the specified digital frequency bins $k$ and thereby the time-domain signal is converted to a frequency-domain signal by applying the basis vector $\psi_{k}=\left[1, e^{-j \frac{2 \pi}{L_{H, m}} k}, \cdots, e^{-j \frac{2 \pi}{L_{H, m}}\left(L_{H, m}-1\right) k}\right]^{\top}$. The integer signal $x_{d_{2}}\left[n_{d_{2}}\right]$ has been lowpassed filtered and downsampled as described in Sec. III-B. Since the STFT of real signals is symmetric in magnitude and all integer numbers are also real numbers, only the spectral components corresponding to positive frequencies are estimated and the magnitude is multiplied by 2 , as shown in (8). The highest positive frequency after the $2^{\text {nd }}$ decimation stage corresponds to the Nyquist frequency given in (7).

$$
f_{N, d_{2}}=\frac{f_{s, d_{2}}}{2}
$$

Since the set of basis vectors $\left\{\psi_{k}\right\}$ is orthogonal and each element has norm $\sqrt{L_{H, m}}$, the results are normalised by the length of the STFT window $1 / L_{H, m}$ in the analysis formula (8), which considers a rectangular window. Additionally, the estimated amplitude of the $k^{\text {th }}$ spectral component is divided by $\sqrt{2}$ in order to obtain the RMS value.

$$
\begin{aligned}
X_{d_{2}}^{n c}[m ; k] & =\frac{2}{\sqrt{2} L_{H, m}} \sum_{n_{L}=0}^{L_{H, m}-1} x_{d_{2}}\left[m-n_{L}\right] e^{-j \frac{2 \pi\left(m-n_{L}\right)}{L_{H, m}} k}(8) \\
k & \in\left\{0,1, \cdots,\left\lfloor\frac{L_{H, m}}{2}\right\rfloor\right\}
\end{aligned}
$$

The index $m$ indicates the latest sample of the sliding window as shown in (9a) and $\Delta f_{m}$ corresponds to the frequency resolution of the discrete STFT calculated in (9b) at sample index $m$. The parameter $B_{H, 0}$ in (9a) refers to the number 
of samples of overlap of the sliding window at sample index $m=0$.

$$
\begin{aligned}
m & =\left\lfloor\frac{n_{d_{2}}}{L_{H, 0}-B_{H, 0}}\right\rfloor\left(L_{H, 0}-B_{H, 0}\right) \\
\Delta f_{m} & =\frac{f_{s, d_{2}}}{L_{H, m}}
\end{aligned}
$$

In transmission line protection the fundamental component $f_{1, m}$ of the voltage and current signals is of major concern and corresponds to the power system frequency. The estimated frequency $f_{1, m}$ is calculated later in (15). Nevertheless, in case of the DSPU for transformer protection the $2^{\text {nd }}$ to $5^{\text {th }}$ harmonics are of interest in order to detect inrush and overexcitation situations [12]. Since synchronised phasors are published by the harmonic module, it is important that the accumulated phase delays $\gamma_{k, d_{i}}$ and the accumulated amplitude errors of the $k^{\text {th }}$ spectral component, introduced by the preceding FIR filters after the $i^{\text {th }}$ decimation stage, are properly compensated. The individual amplitude errors $e_{k, i}$ in p.u., introduced by the $i^{\text {th }}$ FIR filter, are obtained from the frequency response in Fig. 3. The accumulated phase delay $\gamma_{k, d_{i}}$ is calculated in (10) and the amplitude compensation factor $E_{k, d_{i}}$ is obtained by (11). If the group delay and amplitude error of the analog anti-aliasing filters are known, they should be included as well in (10) and (11), respectively.

$$
\begin{aligned}
\gamma_{k, d_{i}} & =2 \pi f_{k, m} \sum_{j=1}^{i} \tau_{j} \\
E_{k, d_{i}} & =\prod_{j=1}^{i} \frac{1}{1-e_{k, j}}
\end{aligned}
$$

Using $\gamma_{k, d_{i}}$ and $E_{k, d_{i}}$, the estimate $X_{d_{2}}^{n c}[m ; k]$ in (8) is compensated in (12).

$$
X_{d_{2}}[m ; k]=E_{k, d_{i}} X_{d_{2}}^{n c}[m ; k] e^{j \gamma_{k, d_{2}}}
$$

Then the phase and amplitude compensated spectral estimate $X_{d_{2}}[m ; k]$ can be labelled by an appropriate time stamp of a point in time defined in (13).

$$
t_{H, m}=\frac{m}{f_{s, d_{2}}}-\sum_{j=1}^{i} \tau_{j}
$$

According to IEEE standard C37.118.1-2011 [16] the time tag is supposed to be placed in the middle of the observation window. Nonetheless, there is no standard for phasor quantities used by protection functions in substations, since these are traditionally part of the protection algorithm itself. From (13) it can be seen that the timestamp has been placed at the beginning of the observation window corresponding to the latest sample $m$. Since the fundamental power system frequency is not always stationary and varies around its nominal value of 50 or $60 \mathrm{~Hz}$, respectively, the length of the observation window $L_{H, m}$ is decreased or increased if the frequency increases or decreases, respectively. Thereby the spectral leakage is
TABLE III

CONFIGURATION OF DSPU

\begin{tabular}{c|c|c|c|c|c|c}
$L_{H, 0}$ & $B_{H, 0}$ & $P_{H}$ & $f_{s, d_{2}}$ & $f_{1,0}$ & $\Delta f_{0}$ & $F_{S}$ \\
\hline \hline 100 & 95 & $1 \mathrm{~ms}$ & $5 \mathrm{kHz}$ & $50 \mathrm{~Hz}$ & $50 \mathrm{~Hz}$ & $1 \mathrm{MHz}$
\end{tabular}

minimized. This is especially important for protection systems close to generator stations where the frequency can vary to a larger extend. The equation (14) shows the adjustment of the window length $L_{H, m}$ to the closest integer, as proposed in [22].

$$
L_{H, m}=\left\lfloor\frac{f_{s, d_{2}}}{f_{1, m}}+0.5\right\rfloor
$$

The power system frequency $f_{1, m}$ is estimated according to the equation in (15), as proposed in [23]. In this frequency estimation approach the angle $\theta$ of the positive sequence phasor is used. The constant cycle time of the harmonic module $P_{H}$ is calculated in (16).

$$
\begin{aligned}
f_{1, m} & =\frac{\theta[m]-\theta[m-1]}{2 \pi P_{H}} \\
P_{H} & =\frac{L_{H, 0}-B_{H, 0}}{f_{s, d_{2}}}
\end{aligned}
$$

There are other methods to reduce the spectral leakage in case of off-nominal fundamental frequency, such as the adjustment of $F_{S}$ of the ADC [24]. Nevertheless, this is not applicable in most CPC architecture, since all the ADCs are synchronised to an external absolute time reference. Minimizing spectral leakage can also be achieved through an enhanced interpolated-DFT [25]. Nonetheless, this approach has the disadvantage that a minimum window length of two fundamental periods has to be used and thus decreases the time location of the signal. Since the speed requirement in power system protection is of high importance, a one cycle observation window is desirable.

\section{Simulation And Results}

In this section the performance of the discrete STFT is verified under step changes in phase and magnitude as well as during a frequency ramp test. Lastly, the harmonic module is tested by applying the 3-phase signal of a corresponding single-phase to ground fault. The processed signals are compensated according to their group delays $\tau_{i}$ in (4) and displayed according to their timestamp $t_{H, m}$ in (13). The DSPU has been configured according to the parameters in Tab. II and in Tab. III.

\section{A. Results of Step Changes in Phase and Magnitude}

Fig. 4 shows the step change in magnitude from 0.5 p.u. to 1 p.u., which is applied at time $0 \mathrm{~s}$. It can be seen that the STFT requires one cycle to settle at the magnitude $\left|X_{d_{2}}[m, 1]\right|$ of 1 p.u. of the fundamental component. During this dynamic phase the TVE increases to $50 \%$. Nevertheless, during steady-state the TVE remains below $1 \%$ as required by [16]. Furthermore, 

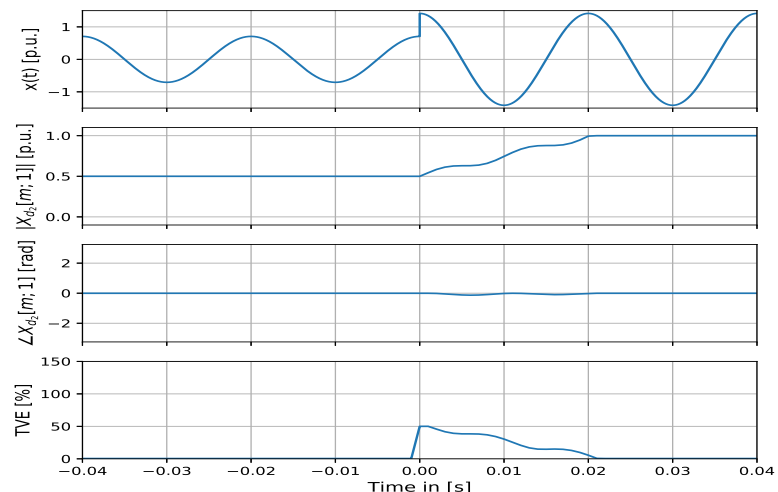

Fig. 4. Performance under a step change in magnitude at time $0 \mathrm{~s}$
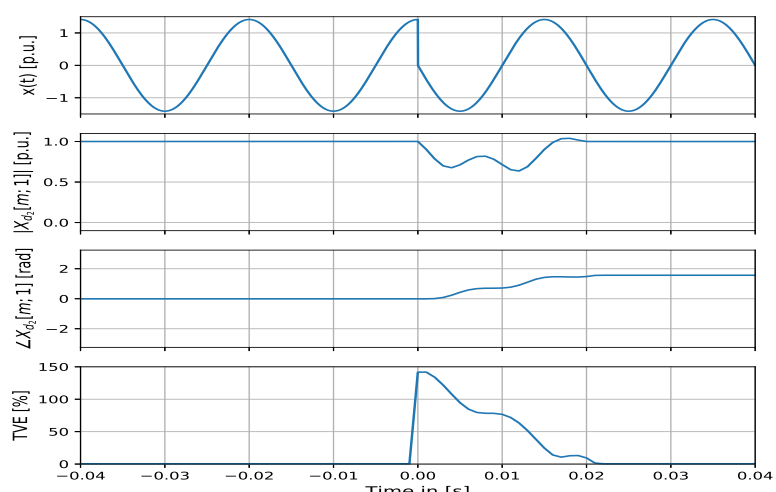

Fig. 5. Performance under a step change in phase at time $0 \mathrm{~s}$

it can be observed that the phase estimation is only marginal affected by the step change in magnitude.

Fig. 5 depicts the step change in phase from 0 to $\frac{\pi}{2}$ at time $0 \mathrm{~s}$. It can be seen that the estimate of the phase $\angle X_{d_{2}}[m, 1]$ settles at $\frac{\pi}{2}$ after one period. The TVE increases above $100 \%$ during the transient phase and then settles below the required $1 \%$ during steady-state. It can also be seen that the estimate of the magnitude $\left|X_{d_{2}}[m, 1]\right|$ of the fundamental component is shortly affected by the step change in phase.

\section{B. Results of Frequency Ramp Test}

The second test consists of a frequency ramp test as shown in Fig. 6. The frequency ramp rate of $1 \frac{\mathrm{Hz}}{\mathrm{s}}$ is applied at time $0 \mathrm{~s}$ and plotted until time $2 \mathrm{~s}$. It can be seen that the estimated fundamental frequency $f_{1, m}$ is correctly estimated according to (15) and rises with the defined rate of $1 \frac{\mathrm{Hz}}{\mathrm{s}}$. It can also be observed that the length of the observation window $L_{H, m}$ is adjusted to the closest integer value according to (14). Due to the adjustment of the length of the observation window, the estimate of the magnitude $\left|X_{d_{2}}[m, 1]\right|$ and the phase $\angle X_{d_{2}}[m, 1]$ of the fundamental component are kept stable.

\section{Results of Numerical EMT Simulation}

This test applies a secondary three phase current signal $i_{s, 3 \phi}(t)$ to the DSPU algorithms corresponding to a phase A
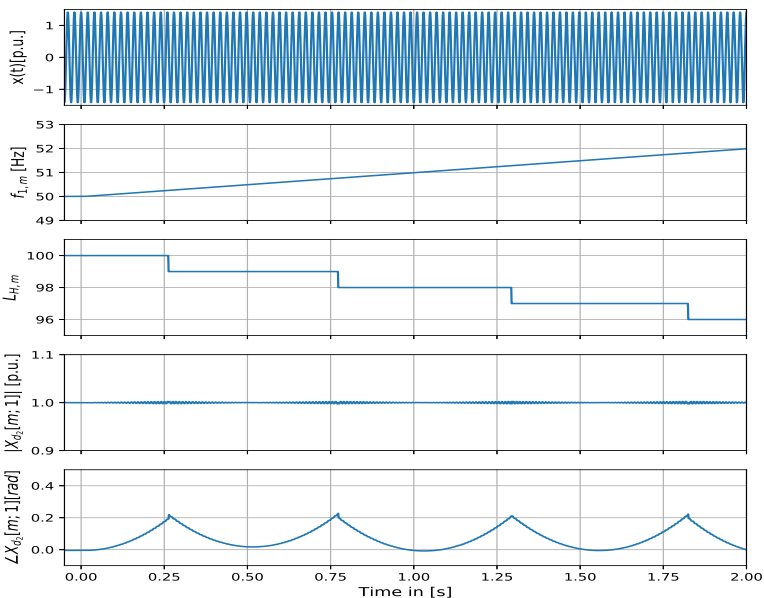

Fig. 6. Performance during ramp of system frequency of $1 \mathrm{~Hz} / \mathrm{s}$

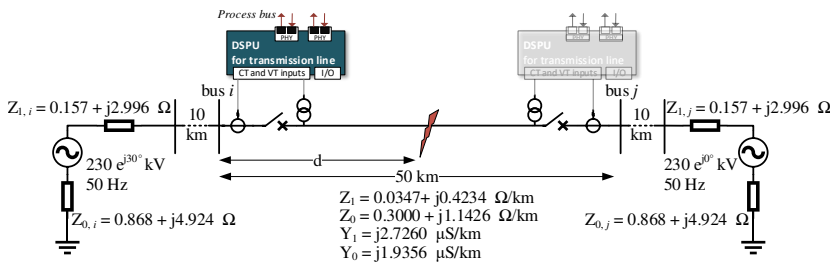

Fig. 7. Single line diagram of the tested three-phase $230 \mathrm{kV}$ power system

to ground fault. The current signal has been obtained by a numerical EMT simulation in PSCAD using the $230 \mathrm{kV}$ power system model shown in Fig. 7. The fault is applied between bus $i$ and bus $j$ at a distance $d$ of $10 \mathrm{~km}$ from bus $i$. The signal $i_{s, 3 \phi}(t)$ has been band-limited by a $2^{\text {nd }}$ order Butterworth filter with a cutoff frequency of $400 \mathrm{kHz}$ representing the analog anti-aliasing filter of the DSPU. This leads to an oversampled discrete-time quantized signal $x_{3 \phi}[n]$ with $F s=1 \mathrm{MHz}$ according to (1). The Fig. 8 shows the current signal and the results of the STFT in terms of magnitude and phase of the phase A current. The phase A to ground fault has been applied at time $0.3 \mathrm{~s}$ and the current is subject to a significant decaying DC offset. It can be seen that the magnitude of the fundamental component $\left|X_{d_{2}}\right|[m, 1]$ of phase A reaches $4 \mathrm{~A}$ at $12 \mathrm{~ms}$ and stabilizes after $20 \mathrm{~ms}$. Moreover, the sharp edges of the magnitude estimates are caused by the frequent changes of the length of the observation window $L_{H, m}$ during the dynamic phase of the fault. At time $0.38 \mathrm{~s}$ the breaker opens at both line ends and the magnitude of the current settles to zero within one power system cycle. Once the magnitude settles below a configured threshold, the discrete STFT algorithm stops estimating the phasor components.

\section{Discussion}

The presented results show that the $1 \mathrm{MHz}$-sampled signals are sufficiently filtered and downsampled in order to be used by the discrete STFT while still complying with the maximum error and minimum attenuation requirements in [15]. This filter 

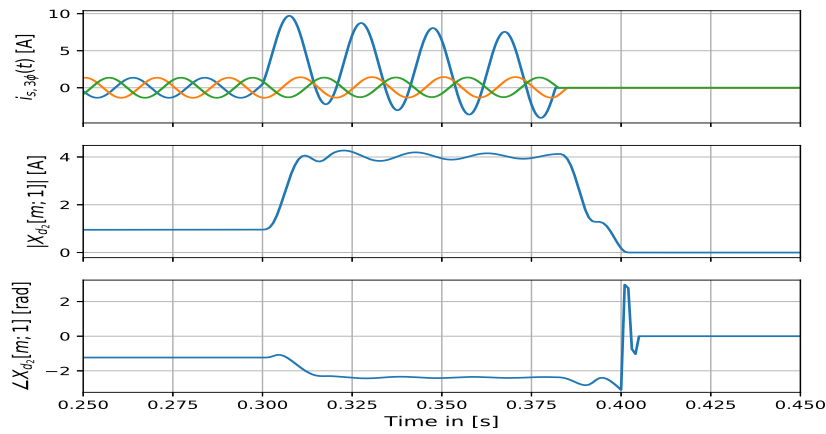

Fig. 8. Performance of STFT during a phase A to ground fault

performance is obtained by a relatively high number of taps $M_{i}$. Due to the existing margin between the requirements and the filter characteristics, it is expected that the number of taps $M_{i}$ can be further reduced in the future. Additionally, it has been shown that the phase delay is properly compensated due to the linear phase characteristics of the passband of the FIR LP. Lastly, the results show that the TVE can be limited to below $1 \%$ during steady-state. Moreover, it can be deduced from the dynamic response of the discrete STFT that different implementations of the DSP algorithm, such as window length or window type, result in different dynamic responses. Therefore it is important in such a CPC architecture that the dynamic performance of the DSP algorithms for substation protection are specified similar to the IEEE standard C37.118 for synchrophasor measurements.

\section{CONCLUSiON}

This paper has proposed a new CPC architecture based on DSPUs that enables the scalable integration of high sampling rate protection applications in digital substations. Furthermore, the design of the transmission line DSPU has been discussed and the structure of its DSP algorithm has been shown. In detail, the design of the multirate filter bank and the discrete STFT have been elaborated and their functional performance has been verified by analysing the behaviour under step changes and during off-nominal frequencies as well as by conducting numerical EMT simulation. As future work, the remaining DSP modules are developed and tested such as travelling-wave protection module, time-domain protection module or interharmonics module, and their corresponding IEC 61850 datasets need to be derived.

\section{REFERENCES}

[1] M. Bollen, S. Rönnberg, F. Zavoda, R. Langella, S. Djokic, P. Cuifo, J. Meyer, and V. Cuk, "Consequences of smart grids for power quality: Overview of the results from cigre joint working group c4.24/cired," in 2017 IEEE PES Innovative Smart Grid Technologies Conference Europe (ISGT-Europe), Sept 2017, pp. 1-6.

[2] "European power system 2040 completing the map - the ten-year network development plan 2018 system needs analysis," ENTSO-E European Network of Transmission System Operators for Electricity, Tech. Rep., 2018.

[3] S. Kariyawasam, A. D. Rajapakse, and N. Perera, "Investigation of using IEC 61850-sampled values for implementing a transient-based protection scheme for series-compensated transmission lines," IEEE Transactions on Power Delivery, vol. 33, no. 1, pp. 93-101, Feb 2018.
[4] E. O. Schweitzer, B. Kasztenny, and M. V. Mynam, "Performance of time-domain line protection elements on real-world faults," in 2016 69th Annual Conference for Protective Relay Engineers (CPRE), April 2016, pp. 1-17.

[5] S. Brahma, "Advancements in centralized protection and control within a substation," IEEE Transactions on Power Delivery, vol. 31, no. 4, pp. 1945-1952, Aug. 2016.

[6] H. F. Albinali and A. P. S. Meliopoulos, "Resilient protection system through centralized substation protection," IEEE Transactions on Power Delivery, vol. 33, no. 3, pp. 1418-1427, June 2018.

[7] D. M. E. Ingram, P. Schaub, R. R. Taylor, and D. A. Campbell, "Network interactions and performance of a multifunction IEC 61850 process bus," IEEE Transactions on Industrial Electronics, vol. 60, no. 12, pp. 59335942, Dec 2013.

[8] G. Kunz, J. Machado, E. Perondi, and V. Vyatkin, "A formal methodology for accomplishing IEC 61850 real-time communication requirements," IEEE Transactions on Industrial Electronics, vol. 64, no. 8, pp. 6582-6590, Aug 2017.

[9] H. D. Ngo and H. S. Yang, "Latency and traffic reduction for processlevel network in smart substation based on high-availability seamless redundancy," IEEE Transactions on Industrial Electronics, vol. 63, no. 4, pp. 2181-2189, April 2016.

[10] N. Jiang, W. Bin, and D. Xinzhou, "Travelling wave sampling data sharing solution in distribution substation based on IEC61850," in 12th IET International Conference on Developments in Power System Protection (DPSP 2014), March 2014, pp. 1-5.

[11] "IEEE recommended practice for signal treatment applied to smart transducers," IEEE Std 21451-001-2017, pp. 1-109, Dec 2017.

[12] F. Hohn, J. Wang, and L. Nordström, "Distributed signal processing units for centralized substation protection and control," in 14th IET International Conference on Developments in Power System Protection (DPSP 2018), March 2018, in press.

[13] IEC 61850-5:2013 Communication networks and systems for power utility automation - Part 5: Communication requirements for functions and device models, TC 57 Std., 2013.

[14] IEC 61869-9:2016 Instrument transformers - Part 9: Digital interface for instrument transformers, TC 38 Std., 2016.

[15] IEC 61869-6:2016 Instrument transformers - Part 6: Additional general requirements for low-power instrument transformers, TC 38 Std., 2016.

[16] "IEEE standard for synchrophasor measurements for power systems," IEEE Std C37.118.1-2011 (Revision of IEEE Std C37.118-2005), pp. 1-61, Dec 2011.

[17] "IEEE guide for protective relay applications to transmission lines," IEEE Std C37.113-2015 (Revision of IEEE Std C37.113-1999), pp. 1141, June 2016.

[18] J. M. Herrera, M. S. Mingarro, S. L. Barba, D. Dolezilek, F. Calero, A. Kalra, and B. Waldron, "Case study of time-domain automation and communications: Field-proven benefits to automation, control, monitoring, and special protection schemes," in 2017 Saudi Arabia Smart Grid (SASG), Dec 2017, pp. 1-8.

[19] "IEEE guide for application of optical instrument transformers for protective relaying," IEEE Std C37.241-2017, pp. 1-50, March 2018.

[20] "IEEE guide for the application of rogowski coils used for protective relaying purposes," IEEE Std C37.235-2007, pp. c1-45, Feb 2008.

[21] T. Parks and J. McClellan, "Chebyshev approximation for nonrecursive digital filters with linear phase," IEEE Transactions on Circuit Theory, vol. 19, no. 2, pp. 189-194, March 1972.

[22] J. P. Benco, F. P. Perfect, D. G. Hart, and J. D. Stoupis, "Generator protection system and method for phasor estimation and frequency tracking during frequency ramping," U.S. patent, Nov. 3, 1998. [Online]. Available: https://patents.google.com/patent/US5832413

[23] A. G. Phadke, J. S. Thorp, and M. G. Adamiak, "A new measurement technique for tracking voltage phasors, local system frequency, and rate of change of frequency," IEEE Transactions on Power Apparatus and Systems, vol. PAS-102, no. 5, pp. 1025-1038, May 1983.

[24] G. Benmouyal, "An adaptive sampling-interval generator for digital relaying," IEEE Transactions on Power Delivery, vol. 4, no. 3, pp. 16021609, Jul 1989.

[25] P. Romano and M. Paolone, "Enhanced interpolated-dft for synchrophasor estimation in fpgas: Theory, implementation, and validation of a pmu prototype," IEEE Transactions on Instrumentation and Measurement, vol. 63, no. 12, pp. 2824-2836, Dec 2014. 